Journal of Knot Theory and Its Ramifications

(C) World Scientific Publishing Company

\title{
IMPOSSIBILITY OF OBTAINING SPLIT LINKS FROM SPLIT LINKS VIA TWISTINGS
}

\author{
MAKOTO OZAWA * \\ Department of Mathematics, School of Education, \\ Waseda University, Nishiwaseda 1-6-1, Shinjuku-ku, \\ Tokyo 169-8050, Japan \\ ozawa@musubime.com
}

\begin{abstract}
We show that if a split link is obtained from a split link $L$ in $S^{3}$ by $1 / n$-Dehn surgery along a trivial knot $C$, then the link $L \cup C$ is splittable. That is to say, it is impossible to obtain a split link from a split link via a non-trivial twisting. As its corollary, we completely determine when a trivial link is obtained from a trivial link via a twisting.
\end{abstract}

\section{Introduction}

Let $L$ be a link in $S^{3}$ and $C$ a trivial knot in $S^{3}$ missing $N(L)$. Then we can get a new link $L^{*}$ in $S^{3}$ as the image of $L$ after doing $1 / n$-Dehn surgery along $C$. We say that $L^{*}$ is obtained from $L$ by an $n$-twisting along $C$. In this paper, we consider the following problem.

Problem. Is it possible that both $L$ and $L^{*}$ are splittable?

For this problem, it is reasonable to make the following definition. An $n$-twisting is said to be non-trivial if $n \neq 0$ and the link $L \cup C$ is non-splittable. Then our result is stated as follows.

Theorem 1. It is impossible to obtain a split link from a split link by a non-trivial twisting.

Next, we consider when a trivial link is obtained from a trivial link by an $n$ twisting. For a trivial knot, this problem has been solved as follows.

*The author was supported in part by Fellowship of the Japan Society for the Promotion of Science for Japanese Junior Scientists. 
Theorem 2. (清, [3]) Suppose that a trivial knot $K^{*}$ is obtained from a trivial knot $K$ by an $n$-twisting along $C$. Then one of the following conclusions holds.

(1) The link $K \cup C$ is a trivial link.

(2) The link $K \cup C$ is a Hopf link.

(3) The link $K \cup C$ is a torus link of type $(4,-2)$ or $(4,2)$, and $n=1$ or -1 respectively.

By Theorems 1 and 2, we obtain the next corollary.

Corollary 1. Suppose that a trivial link $L^{*}=K_{1}^{*} \cup \ldots \cup K_{l}^{*}$ is obtained from a trivial link $L=K_{1} \cup \ldots \cup K_{l}$ by an $n$-twisting along $C$. Then one of the following conclusions holds.

(1) The link $L \cup C$ is a trivial link.

(2) The link $L \cup C$ is a split union of a Hopf link $K_{i} \cup C$ and a trivial link $L-K_{i}$ for some $i \in\{1, \ldots, l\}$

(3) The link $L \cup C$ is a split union of a torus link $K_{i} \cup C$ of type $(4,-2)$ or $(4,2)$ and a trivial link $L-K_{i}$ for some $i \in\{1, \ldots, l\}$, and $n=1$ or -1 respectively.

\section{Preliminaries}

In this section, we prepare some lemmas for Theorem 1. All manifolds are assumed to be compact and orientable, and any srufaces in a 3-manifold are assumed to be properly embedded and in general position.

Let $M$ be a 3 -manifold, and $F_{1}$ and $F_{2}$ two surfaces in $M$. Let $\hat{F}_{1}, \hat{F}_{2}$ be the closed surfaces obtained by capping off $\partial F_{1}, \partial F_{2}$ with disks. Then, for $\alpha \in$ $\{1,2\}$, one defines a graph $G_{\alpha}$ in $\hat{F}_{\alpha}$, where the edges of $G_{\alpha}$ correspond to the arc components of $F_{1} \cap F_{2}$, and the vertices to the components of $\partial F_{\alpha}$. Recall that a 1-sided face in a graph is a disk face with exactry one edge in its boundary.

Recall that if $M$ is a 3-mainfold with torus boundary and $\gamma$ is a slope on $\partial M$, then $M(\gamma)$ denotes the closed maifold obtained by attaching a solid torus $J$ to $M$ so that the boundary of a meridian disk of $J$ has slope $\gamma$ on $\partial M$. Recall also that if $\gamma_{1}, \gamma_{2}$ are two slopes on $\partial M$, then $\Delta\left(\gamma_{1}, \gamma_{2}\right)$ denotes the minimal geometric intersection number of $\gamma_{1}$ and $\gamma_{2}$.

The following lemma will be needed for Theorem 1 .

Lemma 1. Let $M$ be a 3-manifold with torus boundary and let $F_{1}, F_{2}$ be planar surfaces in $M$ with boundary slopes $\gamma_{1}, \gamma_{2}$. Suppose that the graphs $G_{1}, G_{2}$ contain no 1 -sided faces, and that $\Delta\left(\gamma_{1}, \gamma_{2}\right) \geq 1$. Then either the first homology groups $H_{1}\left(M\left(\gamma_{1}\right)\right)$ or $H_{1}\left(M\left(\gamma_{2}\right)\right)$ has a torsion.

Proof. If $\Delta\left(\gamma_{1}, \gamma_{2}\right) \geq 2$, then Lemma 1 follows [1, lemma 2.2]. Otherwise, by [2, Proposition 2.0.1], $G_{1}$ contains a Scharlemann cycle or $G_{2}$ represents all $\left\{1, \ldots,\left|\partial P_{1}\right|\right\}$ types. In the formar case, $M\left(\gamma_{2}\right)$ has a lens space as a connected summand. In the latter case, by [5, Theorem], $H_{1}\left(M\left(\gamma_{1}\right)\right)$ has a torsion. This completes the proof of Lemma 2. 


\section{Proof of Theorem 1}

Suppose that a split link $L^{*}$ is obtained from a split link $L$ by a non-trivial $n$ twisting along $C$. Let $S$ and $S^{*}$ be the splitting spheres for $L$ and $L^{*}$ respectively. Put $M=S^{3}-\operatorname{int} N(C)$. We may assume that $C$ intersects $S$ and $S^{*}$ transversely in the 3 -spheres $M(1 / 0)$ and $M(1 / n)$ respectively, and assume that $|C \cap S|$ and $\left|C \cap S^{*}\right|$ are minimal among all 2-spheres isotopic to $S$ and $S^{*}$ respectively. Then, since $L \cup C$ and $L^{*} \cup C$ are non-splittable, $|C \cap S|$ and $\left|C \cap S^{*}\right|$ are not equal to zero. Put $P_{1}=S-\operatorname{int} N(C)$ and $P_{2}=S^{*}-\operatorname{int} N(C)$. Then by the minimality of $|C \cap S|$ and $\left|C \cap S^{*}\right|$ and by the irreducibility of $M-L, P_{1}$ and $P_{2}$ satisfy the hypothesis of Lemma 2. Hence $H_{1}(M(1 / 0))$ or $H_{1}(M(1 / n))$ has a torsion, this is impossible.

\section{Acknowledgement}

The author would like to thank Prof. Chuichiro Hayashi for his helpful comments.

[1] C. McA. Gordon and J. Luecke, Only integral Dehn surgeries can yield reducible manifolds, Math. Proc. Camb. Phil. Soc. 102 (1987) 94-101.

[2] C. McA. Gordon and J. Lueke, Knots are determined by their complements, J. Amer. Math. Soc. 2 (1989) 371-415.

[3] M. Kouno, K. Motegi and T. Shibuya, Twisting and knot types, J. Math. Soc. Japan 44 (1992) 199-216.

[4] Y. Mathieu, Unknotting, knotting by twists on disks and Property $(P)$ for knots in $S^{3}$, Knots 90 (ed Kawauchi, A), Proc. 1990 Osaka Conf. on Knot Theory and Related Topics, de Gruyter, (1992) 93-102.

[5] W. Parry, All types implies torsion, Proc. Amer. Math. Soc. 110 (1990) 871-875. 